\title{
Cerebral Microvascular Erdheim-Chester Disease: A Perivascular Hematopoietic Vasculopathy
}

\author{
Michael Moussouttas $^{\mathrm{a}}$ Shanu Roemer ${ }^{\mathrm{b}}$ Dennis W. Dickson ${ }^{\mathrm{b}}$ \\ ${ }^{a}$ Cerebrovascular Division, Department of Neurology, Rutgers Robert Wood Johnson Medical School, \\ New Brunswick, NJ, USA; b Neuropathology Laboratory, Department of Neuroscience, Mayo Clinic, \\ Jacksonville, FL, USA
}

\section{Keywords}

Erdheim-Chester disease - Cerebral vasculature .

Microvascular infiltrates . Perivascular microangiopathy .

Infarction · Hemorrhage · Autopsy

\section{Abstract}

Erdheim-Chester disease (ECD) is a rare and elusive hematopoietic malignancy that may involve the nervous system in various ways. Cerebrovascular ECD involves the perivascular infiltration and compromise of any cervicocranial vessel by transformed proliferating histiocytes. Presented is the novel case of a patient with pathologically proven perivascular microangiopathy, manifesting in multifaceted fashion with ischemia, hemorrhage, mass lesions, and edema.

(C) 2021 S. Karger AG, Basel

\section{Case Report}

A 64-year-old man with hypertension, diabetes, hyperlipidemia, and non-Hodgkin's B-cell lymphoma in remission presented with acute onset dysarthria and hemi-paresis/hypesthesia. Head CT was normal, and CTA of the head/neck revealed only minor cervical carotid plaques. Tissue plasminogen activator was administered for presumed acute cerebral ischemia. Clinical deterioration prompted repeat $\mathrm{CT}$ that revealed multiple acute punctate hemorrhages (Fig. 1a, b), for which cryoprecipitate was administered. Systems review indicated fatigue/malaise, arthralgias, abdominal pain, and urinary incontinence. In hospital, the patient experienced recurrent transient neurologic symptoms, a complex partial seizure, and two fevers $>38^{\circ} \mathrm{C}$, for which chest X-rays, urinalyses, and cultures were unrevealing. Cerebral MRI revealed the hemorrhages plus separate acute punctate infarcts (Fig. 1c, d). Trans-thoracic/esophageal echocardiograms were normal.

Review of the serologic, CSF, radiologic, and histologic investigations performed during this (and the later) hospitalization is presented in Table 1. Positive findings included elevated fibrinogen $577 \mathrm{mg} / \mathrm{dL}$, ESR $83 \mathrm{~mm} / \mathrm{h}, \mathrm{CRP} 28 \mathrm{mg} / \mathrm{L}$, and $\beta 2$-microglobulin $3.3 \mathrm{mcg} / \mathrm{mL}$. Immunoglobulin subclass levels were low: IgM 26 $\mathrm{g} / \mathrm{L}$, IgA $54 \mathrm{~g} / \mathrm{L}$, and IgG $321 \mathrm{~g} / \mathrm{L}$. Based on the constitutional symptoms, recurrent fevers, serologic evidence of inflammation, recurrent neurologic symptoms, and new punctate ischemic foci on repeat MRI, the decision was made to pursue angiography and possibly biopsy. Cervicocranial angiography demonstrated no evidence for arterial or venous vasculitis or vasculopathy, and cerebral biopsy revealed no inflammatory, infectious, or neoplastic processes.

Four months later, due to persistent fevers and recurrent neurologic symptoms, the patient was readmitted, and cerebral MRI with contrast revealed circular enhancing lesions (Fig. 1e, f) with nodular widening of the infundibulum. CSF cytology was negative for malignant cells, but noted abundant macrophages/monocytes. Cytometry was negative for atypical lymphocytes. Repeat biopsy karger@karger.com www.karger.com/ced (c) 2021 S. Karger AG, Basel

Karger!
Correspondence to:

Michael Moussouttas, moussouttas@ hotmail.com 


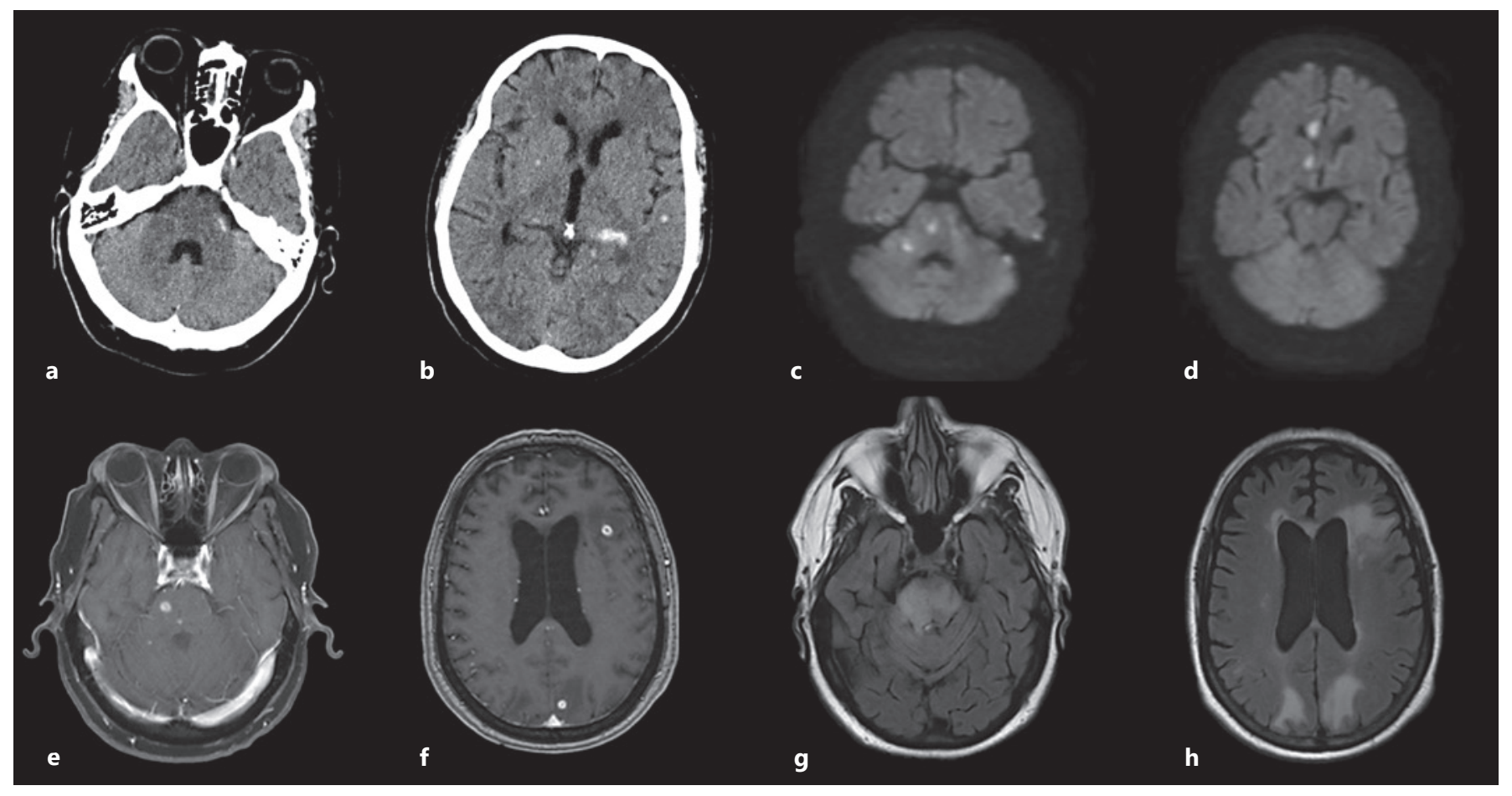

Fig. 1. CT and MR imaging illustrating initial hemorrhagic and ischemic lesions, and later development of mass lesions and cerebral edema. a, b CT demonstrating linear pontine and multifocal subcortical hemorrhages post-tPA. c, d Diffusion MRI revealing several acute punctate infarcts separate from areas of hemorrhage. e, $\mathbf{f}$ Contrast MRI sequences demonstrating several circular enhancing nodular mass lesions. g, h FLAIR imaging revealing extensive edema in the pons and subcortical hemispheric regions. revealed no evidence of an inflammatory, infectious, or neoplastic process.

Later MRI revealed evolving confluent edema in the posterior fossa and in the subcortical hemispheric regions bilaterally (Fig. 1g, h). The patient continued to experience recurrent fevers and progressive neurologic deterioration, with decline in mentation, vision, articulation, and gait, and expired several months later.

\section{Neuropathology}

Histology demonstrated inflammatory cells along the convexity and basal leptomeninges composed of foamy histiocytes and T-cells. The histiocytic infiltration was also present in the thalamus and posterior fossa structures. The pituitary infundibulum had extensive perivascular infiltration with foamy histiocytes (Fig. 2a) that were positive for CD68 (Fig. 2b) and CD163 (Fig. 2c) but negative for S100 (Fig. 2d). CD3-positive T-cells accompanied the histiocyte infiltrate (Fig. 2 e), with $<50 \%$ being positive for the proliferative marker Ki67 (not shown). Stains for B-cell CD20 were negative (Fig. 2f). Subependymal microvasculature had extensive infiltration of perivascular spaces by CD68 histiocytes (Fig. $2 \mathrm{~g}$ ) and CD3 T-cells (Fig. 2h). Pathologic findings were indicative of chronic meningoencephalitis and perivasculitis consistent with the non-Langerhans lymphohistiocytosis Erdheim-Chester disease (ECD). Additionally, multiple subacute infarctions were seen in the temporal lobe (Fig. 2i), thalamus, and posterior fossa structures, in which the histiocytes showed positivity with CD68 (Fig. 2j), but no reactivity for CD163 (Fig. 2k), and minimal reactivity for $\mathrm{S} 100$ positivity (Fig. 2l).

\section{Discussion}

ECD is a rare, acquired, non-Langerhans histiocytic neoplasm associated with autoimmunity [1]. It is linked to somatic mutations of the MAPK pathway (usually BRAF proto-oncogene V600E) that enhance histiocyte proliferation and survival [2]. ECD results in the infiltration of tissues and organs by transformed histiocytes, and causes injury by inflammation and fibrosis [2]. Biopsy is required to confirm diagnosis [2]. The 
Table 1. Summary of pertinent diagnostic investigations performed

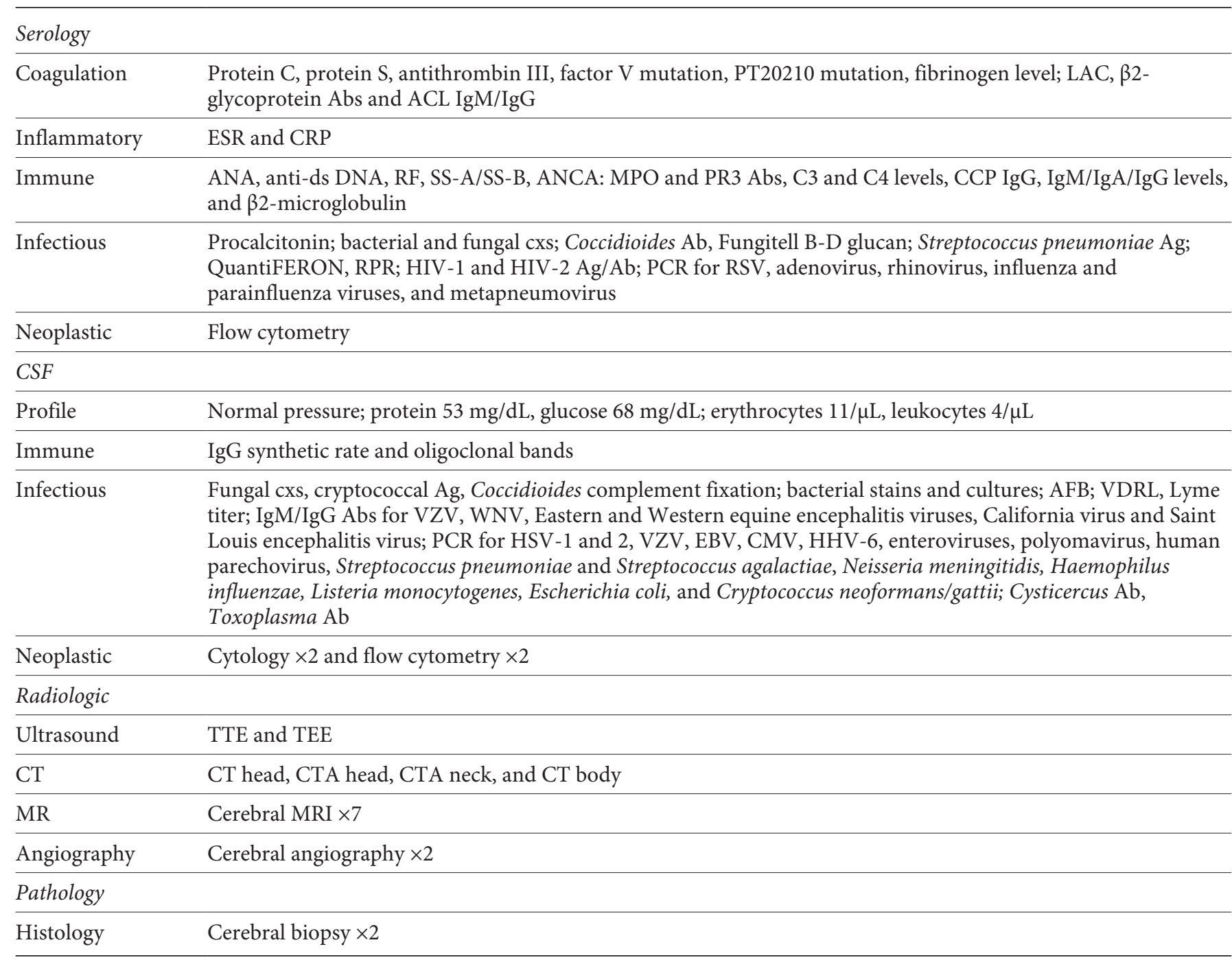

Cxs, cultures.

characteristic features of ECD include diffuse tissue infiltration with CD68-positive and CD163-positive, but S100-negative foamy histiocytes. Dense fibrosis, multinucleated (Touton) cells, and reactive lymphocytosis are often present [3]. Treatment with interferon- $\alpha$ constitutes first-line therapy [3], but prognosis remains guarded, with a $60 \%$ mortality rate 3 years after diagnosis [4].

Commonly involved organs include the bone, skin, eye, pituitary, heart, vessels, lungs, kidneys, liver, spleen, pleura, and retroperitoneum $[3,4]$. Clinical symptoms may include fever, fatigue, bone pain, xanthelasmata, pituitary endocrine dysfunction, and manifestations of car- diac, pulmonary, renal, and vascular compromise $[3,4]$. Cardiac manifestations can include histiocytic invasion of the myocardium and pericardium, at times forming an intra-atrial mass [5]. Perivascular infiltration and fibrosis have been observed in the aortic, coronary, pulmonary, renal, visceral, and iliofemoral arterial systems [5].

CNS involvement occurs in $25-50 \%$ of patients, [3] and may present as alterations in mentation/memory, seizures, cephalalgia, diplopia, dysarthria, dysphagia, hypacousis, sensory-motor deficits, and cranial/peripheral neuropathy $[4,6]$. Imaging may reveal cerebral masses, transverse pontine enhancement, linear ependymal enhancement, pituitary nodules, meningeal lesions, gen- 
Table 2. Conditions associated with cerebrovascular and cervicocranial perivascular leukocytic infiltrates [15-20]

\begin{tabular}{|c|c|c|c|c|c|}
\hline Condition & Inflammatory cell(s) & Vessel type & Vessel size & Vessel locale & CNS focus \\
\hline \multicolumn{6}{|l|}{ Inflammatory } \\
\hline CLIPPERS [15] & Lymphocytes: CD4 > CD8 T-cells & Arteries and veins & Microvascular & Intracranial only & Predominantly pontine \\
\hline $\begin{array}{l}\text { Autoimmune GFAP } \\
\text { astrocytopathy [16] }\end{array}$ & Lymphocytes: CD20 B-cells & Arteries and veins & Microvascular & Intracranial only & Periventricular and subpial \\
\hline Inflammatory CAA [17] & Macrophages: multinucleated giant cells & Arteries & Microvascular & Intracranial only & Cortex and pia \\
\hline Sarcoidosis [18] & Macrophages: epithelioid giant cells & Arteries and veins & $\begin{array}{l}\text { Microvascular and } \\
\text { macrovascular }\end{array}$ & $\begin{array}{l}\text { Intracranial and } \\
\text { extracranial }\end{array}$ & Variable \\
\hline Behcet's disease [19] & Polymorphonuclear cells, lymphocytes, eosinophils, and macrophages & Arteries and veins & $\begin{array}{l}\text { Microvascular and } \\
\text { macrovascular }\end{array}$ & $\begin{array}{l}\text { Intracranial and } \\
\text { extracranial }\end{array}$ & Variable \\
\hline TiPIC (carotidynia) [20] & Lymphocytes & Arteries & Macrovascular & Extracranial carotids & N/A \\
\hline \multicolumn{6}{|l|}{ Proliferative } \\
\hline $\mathrm{ECD}$ & $\begin{array}{l}\text { Macrophages: CD68 and CD163 histiocytes and giant multinucleated } \\
\text { (Touton) cells }\end{array}$ & Arteries & $\begin{array}{l}\text { Microvascular and } \\
\text { macrovascular }\end{array}$ & $\begin{array}{l}\text { Intracranial and } \\
\text { extracranial }\end{array}$ & Variable \\
\hline
\end{tabular}

CLIPPERS, chronic lymphocytic inflammation with pontine perivascular enhancement responsive to steroids; GFAP, glial fibrillary acidic protein; CAA, cerebral amyloid angiopathy; TiPIC, transient perivascular inflammation of the carotid artery; ECD, Erdheim-Chester disease.
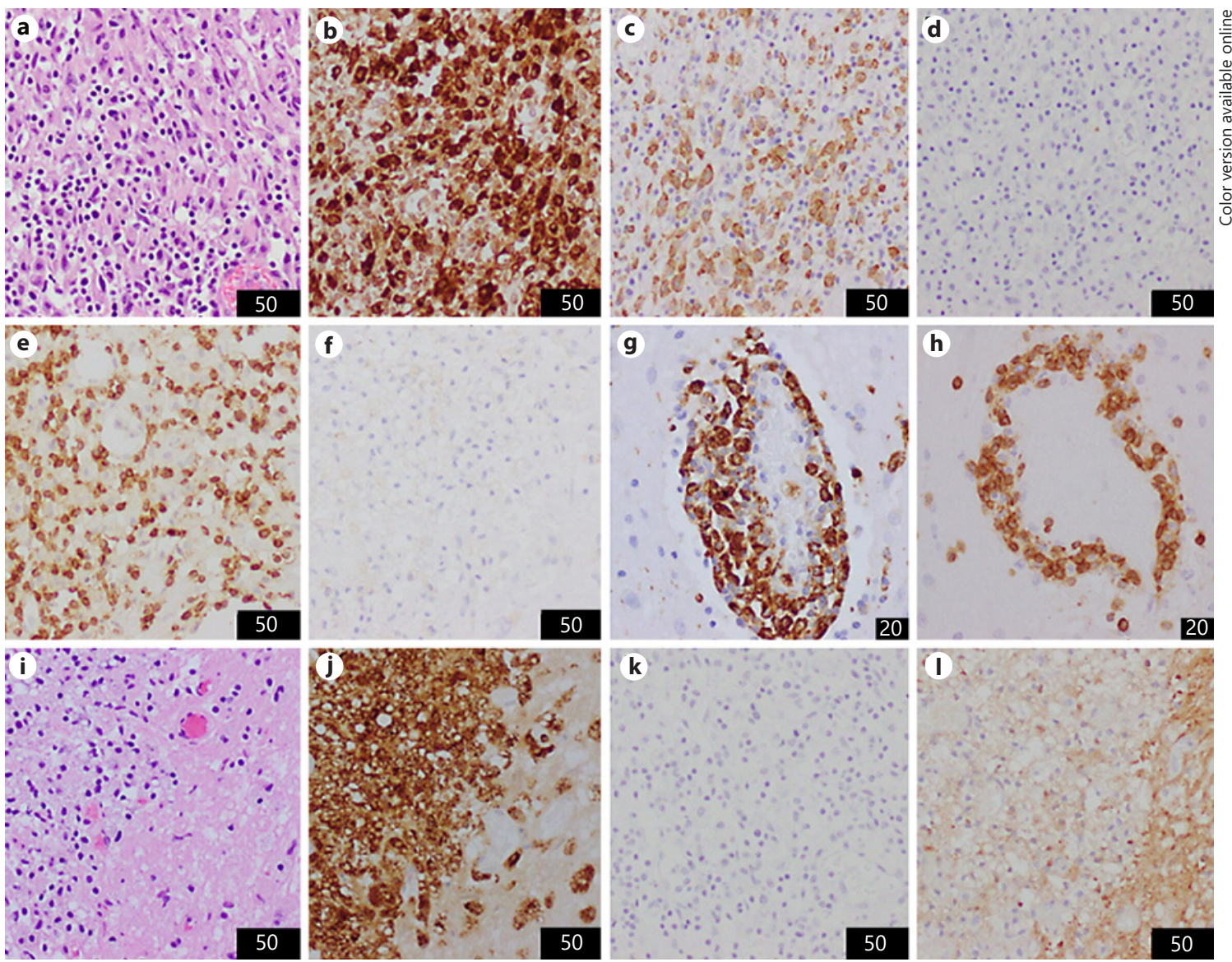

Fig. 2. Histopathology of central nervous system ECD. Hypercellular pleomorphic histiocytic lesion (H\&E) (a), positive for macrophage marker $\mathrm{CD} 68$ (b) and marker for peripheral macrophages (haptoglobulin receptor), CD163 (c), but negative for S100, a Langerhans cell marker (d). e Moderate to marked T-cell infiltrates (CD3) associated with the histiocytes. f No B-cell infiltrates are apparent (CD20). g, h Subependymal vessels have perivascular histiocytes (CD68) and T-cells (CD3). i-I Subacute infarcts. i Histiocyte-rich subacute infarct (H\&E) positive for macrophage-microglial marker CD68 (j) but negative for peripheral macrophage marker CD163 (k) and with sparse S-100 immunoreactivity (I). ECD, Erdheim-Chester disease. 
eralized cerebral atrophy, orbital masses, and craniofacial osteosclerosis [7-9].

Cerebrovascular involvement by ECD is rare and, similar to systemic disease, demonstrates a predilection for the larger cervicocranial vessels. ECD has been associated with perivascular infiltration and stenosis of the cervical carotid [10], intracranial carotid [7], middle cerebral [11], vertebral [12], and basilar [13] arteries, with microvascular involvement implied in the presence of concurrent leukoencephalopathy $[12,14]$. Previous reports documenting perivascular infiltrates of the microvasculature are lacking in ECD. Conditions analogous to ECD that may also demonstrate cervicocranial perivascular leukocyte infiltrates are provided in Table 2.

\section{Conclusions}

In summary, ECD is a rare and elusive hematopoietic malignancy that may involve the nervous system and cerebral vasculature in various ways. We believe this is the first reported case of cerebrovascular ECD that demonstrates the presence of perivascular microangiopathy. Our case also illustrates the multifaceted manner in which ECD may manifest in the central nervous system, including ischemia, hemorrhage, mass lesions, and cerebral edema. Importantly, our case illustrates the difficulty of diagnosing CNS involvement in myeloproliferative disorders, whereby CSF investigations are frequently unrevealing and require serial assessments [21]. As such, reliable biomarkers of CNS ECD are urgently needed to facilitate early diagnosis so as to permit timely initiation of treatment. It is notable that CSF cytology in our patient showed numerous macrophages, which raises the question whether increased S100-negative histiocytes in the CSF may serve as a marker for ECD in patients with CNS involvement. Finally, the dense infiltrate of proliferating subependymal CD3 T-cells raises the theoretical possibility of a secondary autoimmune process, steroid-treated $\mathrm{B}$-cell lymphoma, or that of a secondary malignant $\mathrm{T}$-cell transformation.

\section{Acknowledgements}

We express our gratitude to the patient and relatives who generously donated tissue and clinical records essential to this case report. We also thank Monica Castanedes-Casey and Ariston Librero for their excellent histological assistance.

\section{Statement of Ethics}

This work complies with internationally accepted standards. This research was conducted via the Mayo Clinic neurodegenerative brain bank which operates under an IRB. The study is exempt from Ethics Committee approval as it is a retrospective case report that entailed no active patient interventions.

\section{Conflict of Interest Statement}

The authors have no competing or conflicts of interest to declare.

\section{Funding Sources}

Dr. Dickson is funded by the National Institutes of Health grants: U01 AG046139, UG3 NS104095, and P30 AG062677. The brain bank for neurodegenerative disease is supported by the Rainwater Charitable Foundation, by the Robert E. Jacoby Professorship of Alzheimer's Research, and by the Mayo Medical Foundation.

\section{Author Contributions}

Dr. Moussouttas researched the topic, reviewed the literature, obtained consent, and composed the manuscript. Drs. Roemer and Dickson performed histological evaluations and neuropathologic diagnosis, and assisted in the writing and review of the manuscript.

\section{Informed Consent}

Written informed consent was obtained from family members surviving the patient, who expired, for the collection and compilation of data, and for the publication of this case report and any accompanying images.

\section{References}

1 Roeser A, Cohen-Aubart F, Breillat P, Miyara M, Emile JF, Charlotte F, et al . Autoimmunity associated with Erdheim-Chester disease improves with BRAF/MEK inhibitors. Haematologica. 2019 Nov; 104(11): e502-5.

2 Jacobsen E. Erdheim-Chester disease [Internet]. Alphen aan den Rijn, Netherlands:
Wolters Kluwer; 2020 [updated 2020 Feb 25: cited 2020 Oct 15]. Available from: www.uptodate.com.

3 Diamond EL, Dagna L, Hyman DM, Cavalli G, Janku F, Estrada-Veras J, et al . Consensus guidelines for the diagnosis and clinical management of Erdheim-Chester disease. Blood. 2014 Jul 24;124(4):483-92. 
4 Estrada-Veras JI, O’Brien KJ, Boyd LC, Dave RH, Durham B, Xi L, et al . The clinical spectrum of Erdheim-Chester disease: an observational cohort study. Blood Adv. 2017 Feb 14; $1(6): 357-66$.

5 Villatoro-Villar M, Bold MS, Warrington KJ, Crowson CS, Goyal G, Shah M, et al . Arterial involvement in Erdheim-Chester disease: a retrospective cohort study. Medicine. 2018 Dec;97(49):e13452.

6 Bhatia A, Hatzoglou V, Ulaner G, Rampal R, Hyman DM, Abdel-Wahab O, et al . Neurologic and oncologic features of ErdheimChester disease: a 30-patient series. Neuro Oncol. 2020 Jul 7;22(7):979-92.

7 Drier A, Haroche J, Savatovsky J, Godenèche G, Dormont D, Chiras J, et al . Cerebral, facial, and orbital involvement in Erdheim-Chester disease: CT and MR imaging findings. Radiology. 2010 May;255(2):586-94.

8 Sedrak P, Ketonen L, Hou P, Guha-Thakurta $\mathrm{N}$, Williams MD, Kurzrock R, et al . ErdheimChester disease of the central nervous system: new manifestations of a rare disease. AJNR Am J Neuroradiol. 2011 Dec;32(11):2126-31.

9 Diamond EL, Hatzoglou V, Patel S, AbdelWahab O, Rampal R, Hyman DM, et al . Diffuse reduction of cerebral grey matter volumes in Erdheim-Chester disease. Orphanet J Rare Dis. 2016 Aug 2;11(1):109.
10 Choi KH, Kim JH, Kim JT, Choi SM, Lee SH, Park MS, et al . Unusual carotid stenosis detected by F-18 FDG PET/CT. Eur Neurol. 2013;69(6):375-6.

11 Fargeot G, Stefanizzi S, Depuydt S, Klapczynski F, Ameri A. Association between ischemic stroke and Erdheim-Chester disease: a case report and review of literature. J Stroke Cerebrovasc Dis. 2017 Aug;26(8):e153-5.

12 Sagnier S, Debruxelles S, Lepreux S, Sibon I. Erdheim-Chester disease: an unusual cause of intracranial vasculitis and progressive leukoencephalopathy. J Stroke Cerebrovasc Dis. 2016 May;25(5):e63-5.

13 Mathis $S$, Godenèche G, Haroche J, Milin S, Julian A, Berthomet A, et al . Long-term outcome of basilar stenosis in Erdheim-Chester disease: a case report. Medicine. 2016 Sep; 95(36):e4813.

14 Chiapparini L, Cavalli G, Langella T, Venerando A, De Luca G, Raspante S, et al . Adult leukoencephalopathies with prominent infratentorial involvement can be caused by Erdheim-Chester disease. J Neurol. 2018 Feb; 265(2):273-84.
15 Dudesek A, Rimmele F, Tesar S, Kolbaske S, Rommer PS, Benecke R, et al . CLIPPERS: chronic lymphocytic inflammation with pontine perivascular enhancement responsive to steroids. Review of an increasingly recognized entity within the spectrum of inflammatory central nervous system disorders. Clin Exp Immunol. 2014 Mar;175(3):385-96.

16 Kunchok A, Zekeridou A, McKeon A. Autoimmune glial fibrillary acidic protein astrocytopathy. Curr Opin Neurol. 2019 Jun;32(3):452-8.

17 Eng JA, Frosch MP, Choi K, Rebeck GW, Greenberg SM. Clinical manifestations of cerebral amyloid angiopathy-related inflammation. Ann Neurol. 2004 Feb;55(2):250-6.

18 Jachiet V, Lhote R, Rufat P, Pha M, Haroche J, Crozier S, et al . Clinical, imaging, and histological presentations and outcomes of stroke related to sarcoidosis. J Neurol. 2018 Oct;265(10):2333-41.

19 Al-Araji A, Kidd DP. Neuro-Behcet's disease: epidemiology, clinical characteristics, and management. Lancet Neurol. 2009 Feb;8(2): 192-204.

20 Upton PD, Smith JG, Charnock DR. Histologic confirmation of carotidynia. Otolaryngol Head Neck Surg. 2003 Oct;129(4):443-4.

21 Scott BJ, Douglas VC, Tihan T, Rubenstein JL, Josephson SA. A systematic approach to the diagnosis of suspected central nervous system lymphoma. JAMA Neurol. 2013 Mar 1;70(3): 311-9. 